\title{
Experiments on soliton motion in annular Josephson junctions
}

\author{
Davidson, A.; Dueholm, B.; Pedersen, Niels Falsig
}

Published in:

Journal of Applied Physics

Link to article, DOI:

10.1063/1.337324

Publication date:

1986

Document Version

Publisher's PDF, also known as Version of record

Link back to DTU Orbit

Citation (APA):

Davidson, A., Dueholm, B., \& Pedersen, N. F. (1986). Experiments on soliton motion in annular Josephson junctions. Journal of Applied Physics, 60(4), 1447-1454. https://doi.org/10.1063/1.337324

\section{General rights}

Copyright and moral rights for the publications made accessible in the public portal are retained by the authors and/or other copyright owners and it is a condition of accessing publications that users recognise and abide by the legal requirements associated with these rights.

- Users may download and print one copy of any publication from the public portal for the purpose of private study or research.

- You may not further distribute the material or use it for any profit-making activity or commercial gain

- You may freely distribute the URL identifying the publication in the public portal

If you believe that this document breaches copyright please contact us providing details, and we will remove access to the work immediately and investigate your claim. 


\title{
Experments on solizon motion in annular Josephson junctions
}

\author{
A. Davidson, a) B. Dueholm, and N. F. Pedersen \\ Physics Laboratory I, The Technical University of Denmark, DK-2800 Lyngby, Denmark
}

(Received 28 March 1986; accepted for publication 2 May 1986)

\begin{abstract}
We report here the results of an extensive experimental investigation of soliton dynamics in Josephson junctions of different annular geometries. The annular geometry is unique in that it allows for the study of undisturbed soliton motion as well as soliton-antisoliton collisons, since there are no boundary effects. We have successfully trapped a single soliton in an annular junction and found good agreement with perturbation theory at low soliton velocity, and evidence of departure from perturbation theory at higher velocity. We also discuss the observation of fine structure on the $I-V$ curve for a single trapped soliton, and evidence linking the stability of the soliton to surface damping.
\end{abstract}

\section{INTRODUCTION}

In the experimental study of solitons in nonlinear systems the long Josephson junction has received an increasing interest over the past years as a model system. In a Josephson junction the physical equivalent of a soliton is a fluxon, one quantum of magnetic flux $\phi_{0}=h / 2 e=2.064 \times 10^{-15} \mathrm{~Wb}$, where $h$ is Planck's constant and $e$ the electron charge. From an experimental point of view this device is attractive, because soliton motion manifests itself in such a way that important information on the soliton dynamics may be extracted from de $I-V$ curve measurements.

Experiments usually use two different one-dimensional geometries: the overlap junction where current is fed perpendicular to the long dimension; and the inline junction with current feed parallel to the long dimension. The current distribution and hence the boundary conditions are different for the two geometries, with the overlap type having a more uniform distribution as opposed to the inline type where the current is confined to the edges.

The theoretical treatment of soliton dynamics has built mainly upon the perturbation theory of McLaughlin and Scott. ${ }^{1}$ Refinements of this theory in the large bias limit were investigated in Refs. 2 and 3. The perturbation theory as given in Ref. 1 has proven quite successful, but does not include boundary effects or soliton-antisoliton interactions in an analytical way. Both of these processes are present in the junction geometries described above and are expected to be of importance. A great deal of work has been done to extend the perturbation theory to include these effects. ${ }^{4.5}$

A new experimental approach is to choose a geometry that has no boundaries at all, i.e., an annular or ring-shaped Josephson junction. ${ }^{6.7}$ This geometry not only eliminates boundary effects, but also, due to flux quantization, makes it possible to trap a single soliton and study the undisturbed motion. These are the closest experimental conditions under which the perturbation theory in its original form is expected to apply.

The paper is organized as follows: In Sec. II we review the perturbation theory with particular emphasis on the relativistic particle picture. The samples and the experimental

a) Permanent address: IBM Research, Yorktown Heights, NY 10598. setup are described in Sec. III and the experimental results are presented in Sec. IV. Finally Sec. V concludes the paper.

\section{PERTURBATION THEORY-THE RELATIVISTIC PARTICLE PICTURE}

It is well known that a lossless infinitely long junction with no driving force (bias current) is described by the sineGordon equation'

$$
-\phi_{x x}+\phi_{t r}+\sin \phi=0 \text {. }
$$

This equation can be solved analytically to yield the single soliton solution $\phi_{s}=4 \tan ^{-1}\left(e^{\xi}\right)$ where $\xi=(x-u t) \gamma(u)$ and $\gamma(u)=1 / \sqrt{1-u^{2}}$ is the Lorentz factor. Here $\phi$ is the phase difference between the two superconducting films. The spatial variable $x$ and time $t$ are normalized with respect to the Josephson penetration depth $\lambda_{J}=\sqrt{\phi_{0} / 2 \pi \mu_{0} d J_{0}}$, and the reciprocal plasma frequency $\omega_{0}^{-1}=1 / \sqrt{2 \pi I_{0} / \phi_{0} C}$, respectively. In these normalizations the soliton velocity $u$ is measured in units of the velocity of light in the barrier, $\bar{c}=\omega_{0} \lambda_{J}$. Here $d$ is the magnetic thickness of the barrier, $I_{0}$ is the critical current, $J_{0}$ the critical current density, and $C$ the junction capacitance. Equation (1) assumes a one-dimensional geometry, i.e., the normalized width $w$ of the junction must satisfy $w<1$.

We now introduce the bias and loss terms in Eq. (1) to get the modified sine-Gordon equation ${ }^{1}$

$$
-\phi_{x x}+\phi_{t t}+\sin \phi=\eta-\alpha \phi_{t}+\beta \phi_{x x t},
$$

where $\eta(x)=J(x) / J_{0}$ is the normalized bias current, $\alpha=1 / \sqrt{\omega_{0} R C}$ is the tunneling loss parameter. $R$ is the normal state resistance, and $\beta$ is the surface loss parameter. In these normalized units the corresponding measurable quantities, junction voltage $v$, and current $i$, are given by $v=\phi_{t}=2 \pi V / \phi_{0} \omega_{0}$, and $i=-\phi_{x}=I / I_{0}$, respectively, where $V$ and $I$ are the unnormalized quantities measured in the laboratory. In an annular junction of length $l$ the normalized relation between voltage and single soliton velocity is given by $v=2 \pi u / l$.

In a perturbation analysis of Eq. (2) McLaughlin and Scott introduced the soliton momentum ${ }^{1}$

$$
p=u \gamma(u)
$$

to get the quasi-relativistic power balance equation 


$$
\frac{d p}{d t}=-\alpha p\left[1+\left(\frac{\beta}{3 \alpha}\right)\left(1+p^{2}\right)\right]+\frac{\pi \eta}{4},
$$

with the steady-state solution

$$
\eta=4 \alpha(p / \pi)\left[1+(\beta / 3 \alpha)\left(1+p^{2}\right)\right]
$$

relating bias $\eta$ and momentum $p$. The expressions given above are subject to certain restrictions. They apply to a single soliton on an infinite line, i.e., boundary collisions and soliton-antisoliton interactions are not taken into account. Also, due to the nature of the perturbation approach, they are not expected to hold if the perturbing terms are too large, i.e., high bias and/or highly damped systems.

In order to relate to physically measurable quantities, we may, by use of Eq. (3), rewrite Eq. (5) to get

$$
\frac{1}{\eta^{2}}=\left(\frac{\pi}{4} \alpha\right)^{2}\left(\frac{1}{u^{2}}-1\right)\left[1+\frac{\beta}{3 \alpha}\left(1-u^{2}\right)\right]^{-2} \text {. }
$$

Keeping in mind that $v=2 \pi u / l$ it is seen from Eq. (6) that a plot of $1 / I^{2}$ vs $1 / V^{2}$ in the case of $\beta=0$ will give a straight line. Deviations from that will be seen for nonzero $\beta$, predominantly at high velocities $(u \rightarrow 1)$. Hence, such a plot provides a convenient method to determine the significance of surface impedance damping.

An interesting feature of Eq. (5) is that it allows for the definition of a set of universal (normalized) force $(\eta / \alpha)$ vs momentum ( $p$ ) curves, if we take $\beta / \alpha$ as a parameter. Figure 1 shows such a plot for various values of $\beta / \alpha$. The effect of the cubic term for high values of $\eta$ is clearly demonstrated. The small circles in the figure show the relevant curve and the range of our experiments.

As mentioned previously a number of important processes and phenomena in real physical systems are not accounted for by the perturbation theory, and consequently a great deal of work has been devoted to refine and extend the theory. The annular junction geometry, on the other hand, is an ideal test vehicie for the simple perturbation model as well as its extensions. The geometries used will be discussed in Sec. III, and we shall conclude this chapter with a few general remarks.

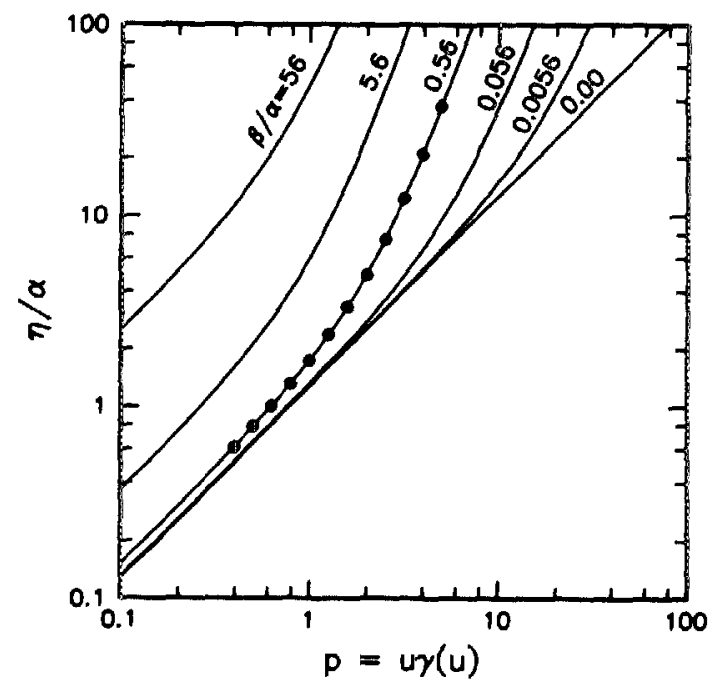

FIG. 1. Perturbation theory: Force vs momentum curves. $\beta / \alpha=$ for each curve is indicated in the figure. The circles fill in the range of data covered by our experiments.
A well known mechanical analog of the long Josephson junction is a chain of elastically coupled pendula. In this model the equivalent of a fluxon or soliton is a $2 \pi$ twist and a spatially uniform bias current corresponds to a uniform torque applied to all the pendula. Figure 2 shows the pendula analog of the annular junction in the case of one trapped soliton. This situation is obtained starting from an open chain, introducing a $2 \pi$ kink and then closing the loop. Once established the soliton can never disappear and additional solitons can only be created in soliton-antisoliton pairs with no net twist (e.g. from a $2 \pi$ twist of a localized region of the chain ).

The creation of a trapped soliton can also occur in a real junction. Like the analog, the annular junction is also a closed loop. If upon cooling through the transition temperature one or more flux quanta are trapped in the junction, they can never disappear as long as the electrodes remain superconducting. Further, since the flux through the ring has to remain constant, additional solitons can only be created in pairs with no net flux.

In the laboratory the junction voltage is measured as a function of bias current. A series of evenly spaced voltage steps are observed, each step corresponding to a discrete number of solitons and antisolitons circulating around the junction. The relativistic particle picture relates the step voltages to the velocity of light in the junction (the maximum velocity of a soliton) by

$$
V_{\text {step }}=(2 \pi n / l) u, \quad u \rightarrow 1,
$$

where $n$ is the total number of solitons and antisolitons and $l$ is the normalized length (circumference) of the junction. For the annular junction $n$ satisfies the relation $n=n_{0}+2 i$, where $i=0,1,2, \ldots$, and $n_{0}$ is the number of solitons initially trapped. With $n_{0}=1$ (one trapped soliton) the odd series of voltage steps is expected while $n_{0}=0$ (no trapped soliton) produces the even series. If the junction has trapped solitons $\left(n_{0}>0\right)$ an infinitely small bias current (force) will cause the solitons to move, which in turn will give a nonzero voltage across the junction. In other words, if trapped solitons are present, the supercurrent should ideally be absent. In a real junction, however, this condition need not be completely satisfied. An imperfection on the ring may act as a pinning site or potential well. With the soliton initially at rest in such a well, a certain force (bias) is required to initiate the motion, resulting in an experimentally measurable, though often small, supercurrent. The influence of such localized imperfections is yet another phenomenon not accounted for by Eq. (5). (See Ref. I for a discussion.)

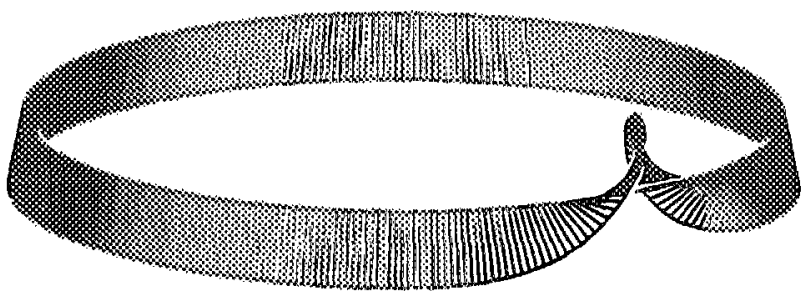

FIG. 2. Computer-generated equivalent pendulum array with a $2 \pi$ kink. $\alpha=0.02, \beta=0.01, \eta=0.4$, and $l=8$. 


\section{SAMPLES AND EXPERIMENTAL SETUP}

In the experiments we have employed two different junction geometries, one developed at Physics Laboratory I, Lyngby, Denmark, and the other at IBM, Yorktown Heights, New York. In both cases the junctions had Nb base electrodes, a tunnel barrier grown by if plasma oxidation and a $\mathrm{Pb}$ or $\mathrm{Pb}$-alloy counterelectrode. Figure 3 shows a micrograph and a schematic of the Lyngby geometry. The $\mathrm{Nb}$ was deposited by dc magnetron sputtering and the top electrode by thermal evaporation. The circumference was approximately $1000 \mu$ and the width $25 \mu$. Current densities ranged between 1 and $100 \mathrm{~A} / \mathrm{cm}^{2}$ with corresponding normalized lengths between 2 and 20. The IBM junctions, as shown in Fig. 4, were edge junctions and both electrodes were electron beam evaporated. ${ }^{8}$ The circumference for these junctions was also close to $1000 \mu$, but the width only $0.3 \mu$. The nominal current density was about $4000 \mathrm{~A} / \mathrm{cm}^{2}$. The edge geometry introduces corrections to the usual formula for the effective $\lambda_{J}$ and taking these into account the result for the IBM edge junctions was a normalized length of about 30 . The whole structure was placed above a superconducting ground plane, and the chips were mounted so that they were aligned with a superconducting loop a few microns above the chip surface, in order to efficiently apply a magnetic field.

The experiments on either junction type were done in separate but similar setups. The samples were placed on a temperature controlled stage in a vacuum can. The Dewars were magnetically shielded and experiments done in a shielded environment. All data were recorded using lownoise preamplifiers (in some cases a lock-in amplifier) then digitized and stored by a precise $A / D$ converter and a microcomputer. To further reduce the noise level, digital signal processing techniques were used.

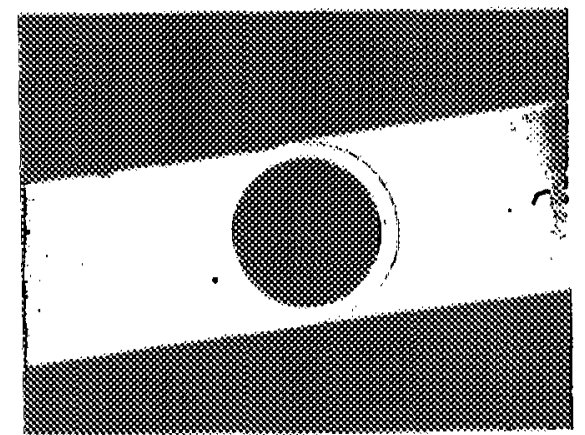

(a)

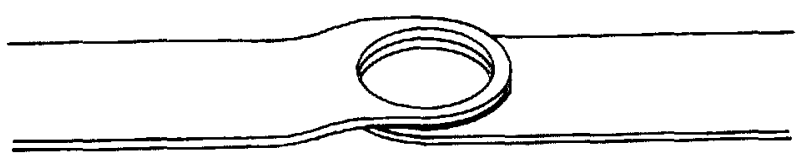

(b)

FIG. 3. Geometry of the junctions fabricated in Lyngby.
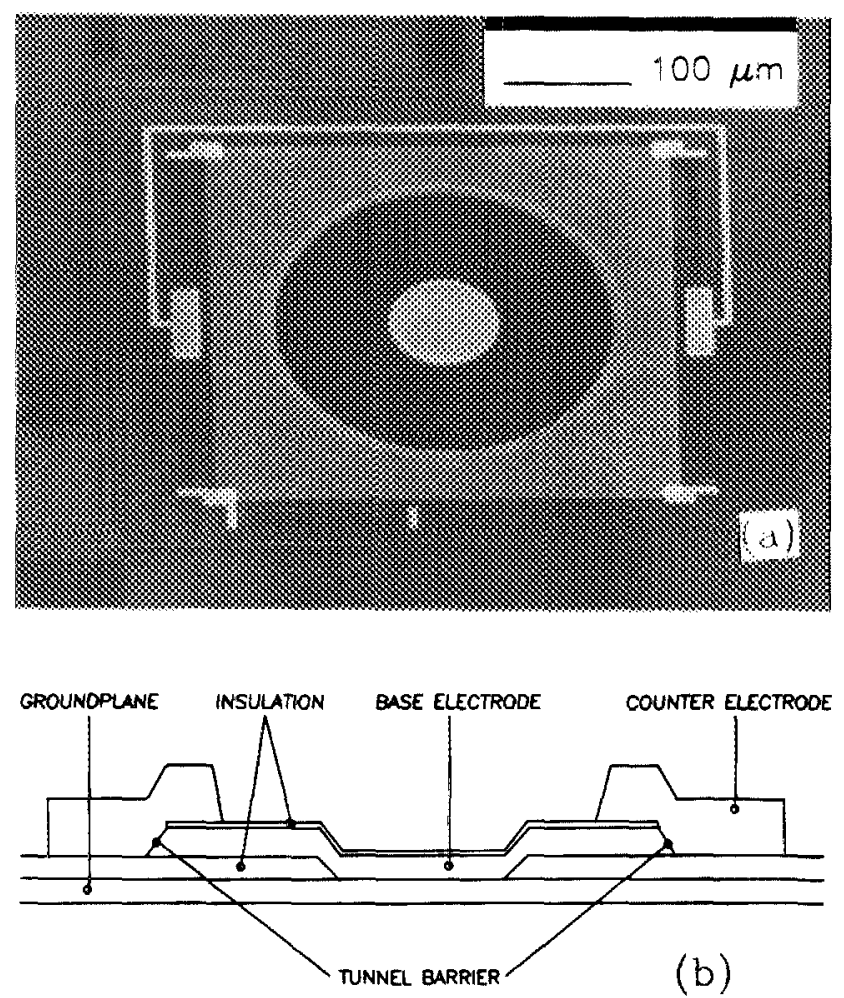

FIG. 4. Geometry of the junctions fabricated at IBM.

\section{EXPERIMENTAL RESULTS}

Common to both of our geometries was a remarkabiy uniform current distribution. Typically the measured critical currents were $0.5-0.6$ times the quasiparticle step at the gap voltage which is close to the values obtained for small $\mathrm{Nb} / \mathrm{Pb}$ junctions. This result may not be too surprising in the case of the IBM geometry, since this design was highly symmetric and care was taken to ensure a homogeneous current feed by means of a resistor network. For the Lyngby geometry, however, we found this observation surprising. Even more surprising was the fact that we never saw evidence for trapped solitons in the IBM junctions. ${ }^{6}$ We are at this point not able to produce any satisfactory explanation for this. In fact we expected that it would prove very difficult to avoid soliton trapping. Even a careful cooling through the $\mathrm{Nb}$ transition temperature (i.e., with base-electrode and ground plane superconducting) followed by application of a magnetic field via the input coil and subsequent cooling through the $\mathrm{Pb}$ transition temperature never resulted in trapped solitons. We shall, however, quote a few results obtained with this geometry and then devote the remainder of this paper to the other geometry in which we have successfully trapped solitons in one sample. Throughout the paper we shall use the notation $(p, q)$ to identify a step, where $p$ and $q$ are the number of solitons and antisolitons present on the line, respectively.

Figure 5 shows the lowest voltage step observed with the IBM geometry. The asymptotic voltage is that expected from a single soliton-antisoliton pair, i.e., a $(1,1)$ step. Also shown in the figure are the results of the perturbation theory [Eq. (5) with $\beta=0$ ] and a simulation. ${ }^{6}$ The theories have 


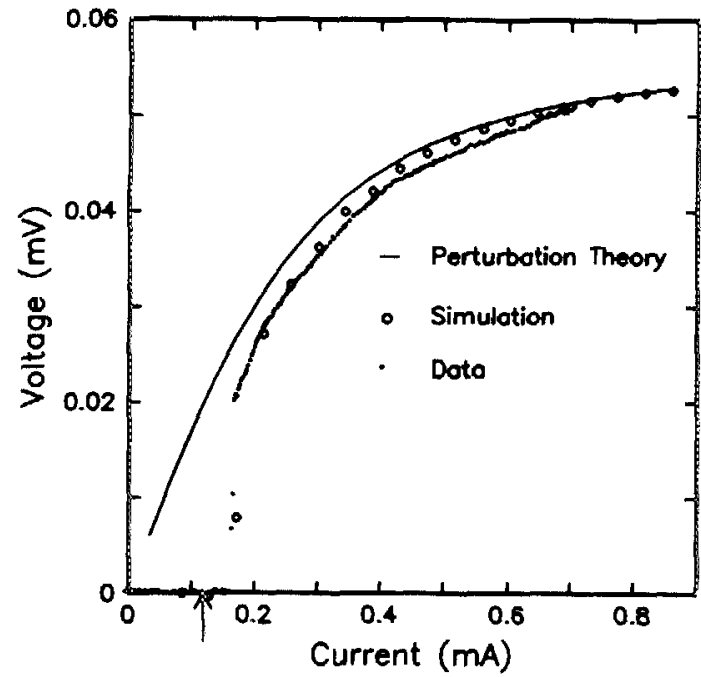

FIG. 5. Experimental $I-V$ curve together with perturbation theory and numerical simulation for the IBM junction $(1,1)$ step.

been fitted to obtain the observed asymptotic voltage. As can be seen the data and the models all merge at high velocities. There is an overall excellent fit between simulation and data, whereas the perturbation theory deviates for low bias. We believe that this deviation is due to soliton-antisoliton collisions which tend to slow down the solitons, thereby decreasing the voltage. ${ }^{9}$ Below a certain bias threshold the soliton and antisoliton annihilate and the junction resets to zero voltage. This feature, nicely reproduced by the simulation, is obviously not included in Eq. (5). The annihilation may be included in a more elaborate theory ${ }^{4.5}$ and the result is indicated by the arrow in Fig. 5. As discussed in Refs. 9 and 10 this theoretical threshold is lower than that observed experimentally.

$A 1 / V^{2}$ vs $1 / 1^{2}$ plot for this junction is shown in Fig. 6 . This type of plot is convenient to determine the asymptotic value of the soliton velocity, or equivalently the speed of light in the barrier. One effect of the $\beta$ term is to shift the experimentally observed part of the step away from the asymptote resulting in increased inaccuracy in the value found directly from the $I-V$ curve; higher accuracy can be obtained

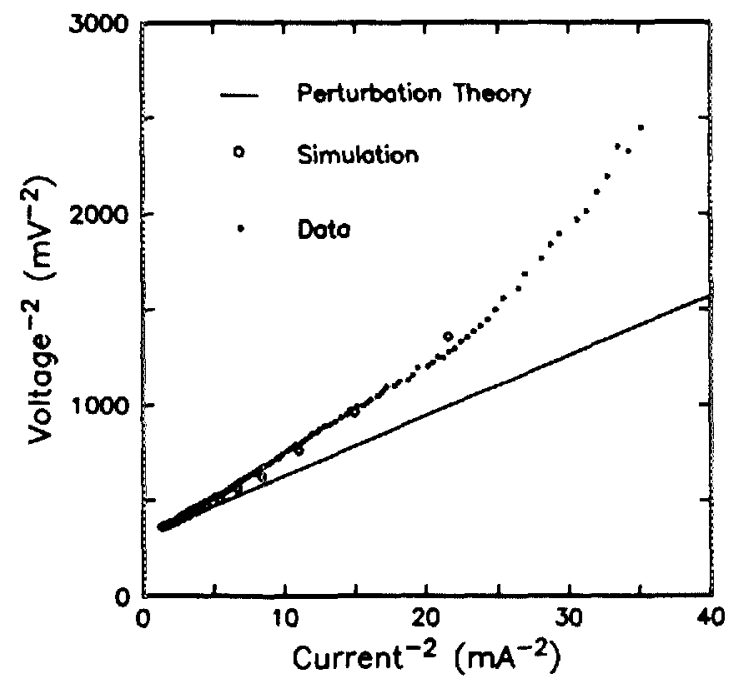

FIG. 6. $V^{-2}$ vs $I^{-2}$ plot corresponding to Fig. 5 . by extrapolating the $1 / V^{2}$ vs $1 / I^{2}$ plot to the $1 / V^{2}$ axis intercept. The experimental data in Fig. 6 tend to a straight line for $I^{-2} \rightarrow 0$, suggesting [Eq. (5)] that $\alpha$ losses are dominant. This is to be expected because of the high current density of the IBM junction. (With increasing current density $\alpha$ losses increase as $\sqrt{J}$ whereas $\beta$ losses remain constant).

Although this junction never trapped a soliton, the behavior in the case of an externally applied magnetic field was as expected. With a field strong enough to modulate the even numbered steps and cause a substantial suppression of the supercurrent, we never observed the odd numbered Fiske steps. ${ }^{11}$ This result supports the prediction that solitons can only be created in pairs in the annular geometry.

In contrast to the IBM junctions, it was straightforward to trap a single soliton with the Lyngby geometry shown in Fig. 3. With no external field applied, and by cooling through the transition temperature in the presence of a small bias current, the trapping of a single soliton readily occurred.

We make a few remarks on soliton trapping in these junctions. We do not know exactly what facilitates or prevents this phenomenon, and what follows are speculations. The main characteristics of the IBM junctions that never trapped a soliton, as opposed to the other geometry which did are: (1) high degree of symmetry, (2) high-quality, very uniform junctions, and (3) the superconducting ground plane. We do not believe that the presence of the ground plane should prevent soliton trapping. On the contrary, when applying the field with the ground plane superconducting, magnetic field lines are forced through the tunnel barrier and thus trapping should be enforced. In these junctions, however, it seems as if some mechanism expels the flux upon subsequent cooling through the transition temperature of the top electrode. The experiments on the junctions with the geometry of Fig. 3 seem to indicate that the losses and/or normalized length also play a role. Several junctions (with rather low current density) of the geometry of Fig. 3 did not trap a soliton, and only one (with $J \cong 60 \mathrm{~A} / \mathrm{cm}^{2}$ ) was really successful. Consequently the geometry itself is not sufficient to ensure trapping.

Figure 7 is strong evidence of soliton trapping for the Lyngby junction with $J=60 \mathrm{~A} / \mathrm{cm}^{2}$. This figure displays the zero-field steps observed from two independent measurements. The even series (thick curves) is associated with a maximum supercurrent of $\$ 3.6 \mathrm{~mA}$, very close to the BCS value. This even series was obtained by very careful cooling through the transition temperature with zero bias current, to ensure that flux was not trapped in the films or the junction. Knowledge of the current density-soliton velocity relation for our junctions (based on numerous other experiments) clearly identifies the low-voltage step as a $(1,1)$ step, i.e., a soliton and an antisoliton traveling in opposite directions on the ring. If we leave a suitable bias current present while cooling, we may obtain the odd series (thin curves in Fig. 7). Here the low-voltage step falls at exactly half the voltage of the $(1,1)$ step and consequently we must interpret this step as being the signature of a single, trapped soliton. Switching between the two series was never observed unless the junction was first warmed through its superconducting critical temperature. 


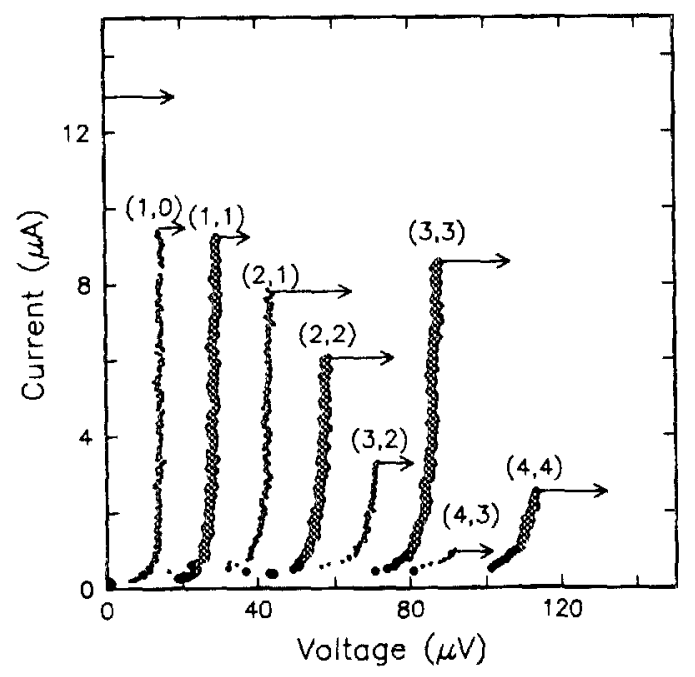

FIG. 7. I-V curve for the Lyngby junction. Thin curves: one soliton trapped. Thick curves: zero solitons trapped.

The odd series is associated with a very small (5\%$10 \%$ ) supercurrent. In accordance with the discussion in Sec. II, we explain this small supercurrent by the existence of pinning sites on the ring. We shall address this question in some detail later.

The $(1,0)$ step has been replotted in Fig. 8 as $1 / V^{2}$ vs $1 /$ $I^{2}$ and fitted to Eq. (6). As can be seen, it is possible to obtain an excellent agreement between the experiments and the perturbation theory by assuming appropriate values of $\alpha$ and $\beta$. Figure 8 clearly demonstrates the effect of the $\beta$ term at high velocities (bias), whereas the low bias data fit a straight line. To illustrate how the $\beta$ term shifts the entire curve we have, for the purpose of comparison, also plotted the case $\beta=0$. Here $\alpha$ has been taken as $\alpha^{\prime}=\alpha+\beta / 3$ which equals the total damping in Eq. (6) for low velocities.

The experiments, data analysis, and fitting proceeded as follows. By use of a germanium resistance thermometer and an automatic temperature controller, we carefully measured the critical current versus temperature. At this point high

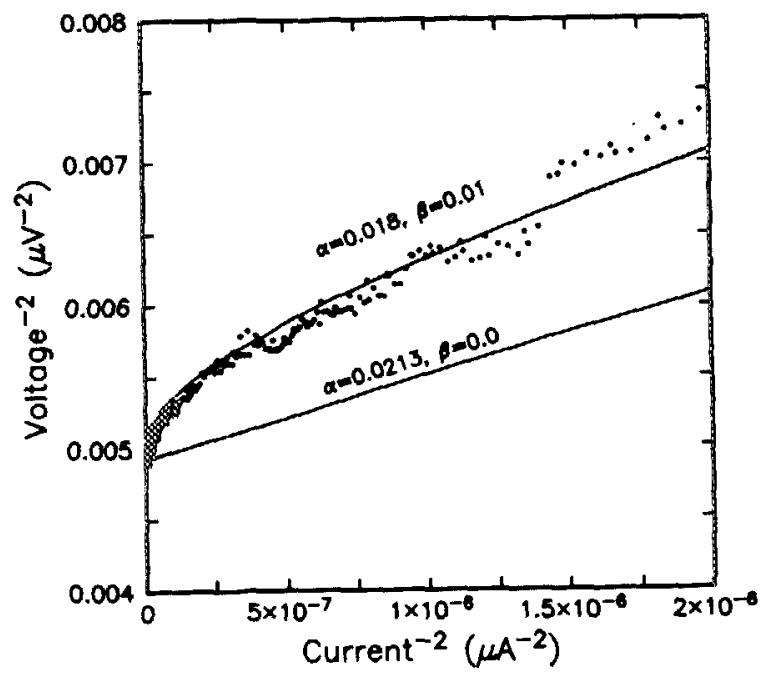

FIG. 8. $(1,0)$ step in a $V^{-2}$ vs $I^{-2}$ diagram. The solid curves are from perturbation theory with the damping parameters shown in the figure. The dots are measured data. accuracy is required since the zero field steps [e.g., the (1.0) step] and the size of the critical current [e.g., the $(0,0)$ step] necessarily have to be obtained from independent measurements. Then the zero-field steps were traced out for different temperatures, in the range from 4.2 to $6.5 \mathrm{~K}$ using digital averaging techniques. Next the data were replotted as shown in Fig. 8 and from this plot we get the speed of light by extrapolation. Finally, by fitting to Eq. (6) the values of $\alpha$ and $\beta$ were extracted. We note that the fitted values of $\alpha$ were consistent with estimates based on bare junction parameters.

The $(1,0)$ step as measured directly is shown in Fig. 9 for a few selected temperatures. In Table I we have summarized the most important parameters obtained from either direct measurement or fitting. We note from this table that the ratio between the current range of the $(1,0)$ step and the critical current (for the zero soliton state) is almost constant (within 4\%). An important result is that, even though the loss parameters $\alpha$ and $\beta$ change by an order of magnitude in this temperature range, we were able to fit all the data with a constant value of $\beta / \alpha$. This result is physically reasonable, since both loss mechanisms depend on the number of quasiparticles available. This number increases with temperature and hence both $\alpha$ and $\beta$ are expected to increase with temperature.

Figure 10 shows a force $(\eta)$ vs momentum $[u \gamma(u)]$ plot for perturbation theory [Eq. (5)] and for a recent numerical simulation ${ }^{12}$ for damping parameters corresponding to Fig. 8. Note that for high values of the bias deviations between Eq. (5) and the simulation occur. In that region perturbation theory ceases to apply, and $u \gamma(u)$ is no longer the momentum. Also in Fig. 10 are shown our experimental results for two assumed values of $\bar{c}$ deviating less than $1 \%$. We conclude that although there is a big sensitivity to the value of $\bar{c}$ used, our experimental results show the deviation from perturbation theory indicated by the numerical simulation.

The constancy of $\beta / \alpha$ means that all of our data can be fitted on a universal force versus momentum curve, as discussed in Sec. II. Consequently we have replotted our data on the $\eta / \alpha$ vs $u \gamma(u)$ plane and the result is shown in Fig. 11 .

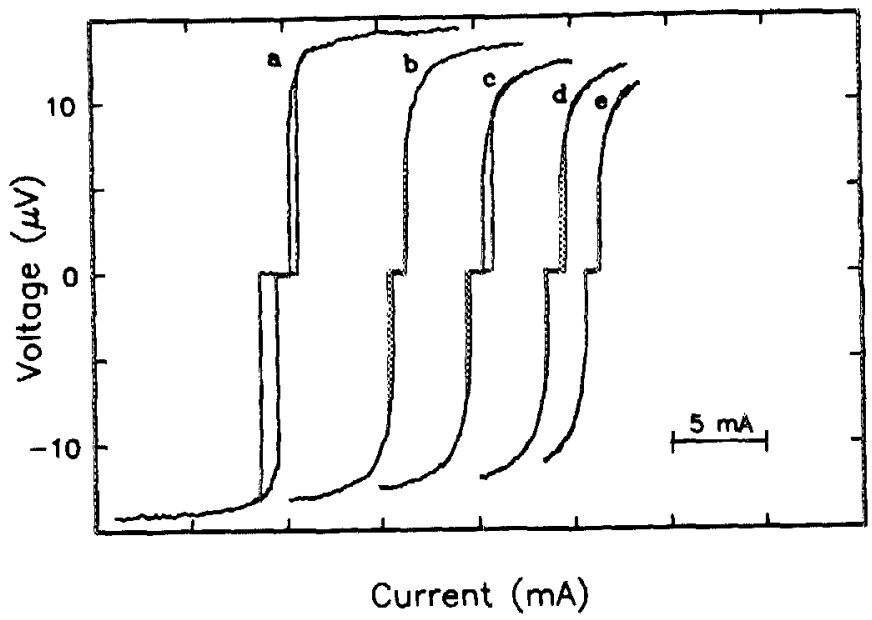

FIG. 9. $(1,0)$ step for 5 temperatures between 4.2 and $6.5 \mathrm{~K}$ (a) $I_{0}=12.7$ $\mathrm{mA}$, (b) $8.56 \mathrm{~mA}$, (c) $6.40 \mathrm{~mA}$, (d) $5.16 \mathrm{~mA}$, and (e) $3.46 \mathrm{~mA}$. 
TABLE I. Measured and fitted parameters at four temperatures for the Lyngby annular junction.

\begin{tabular}{lccccccc}
\hline Data & $\begin{array}{c}I_{0} \\
(\mathrm{~mA})\end{array}$ & $\begin{array}{c}I_{1} \\
(\mathrm{~mA})\end{array}$ & $I_{1} / I_{0}$ & $\begin{array}{c}V_{c} \\
(\mu \mathrm{V})\end{array}$ & $\alpha$ & $\beta$ & $\beta / \alpha$ \\
\hline A352 & 12.7 & 9.1 & 0.72 & 14.32 & 0.018 & 0.01 & 0.56 \\
A354 & 8.56 & 6.2 & 0.72 & 13.71 & 0.055 & 0.0306 & 0.56 \\
A355 & 6.40 & 5.1 & 0.79 & 13.04 & 0.088 & 0.0445 & 0.56 \\
A359 & 3.46 & 2.6 & 0.75 & 12.51 & 0.180 & 0.100 & 0.56 \\
\hline
\end{tabular}

For the three temperatures chosen, we do indeed get an excellent fit to a universal curve with $\beta / \alpha \cong 0.56$, with deviations at high momentum, as expected.

Also shown in Fig. 11 are the supercurrents where we switch from or to zero voltage from the step. The three pairs of lines, $a, b$, and $c$, where this switching occurred are in order from 4.2 to about $6.5 \mathrm{~K}$. Notice that in curve $c$ the hysteresis has nearly disappeared.

The fact that $\beta / \alpha$ is temperature independent also indicates that the cubic dependence in our data stems from surface impedance losses and not from a cubic part in the quasiparticle losses. To our knowledge these results constitute the first measurements on a relativistic particle in a solid-state system.

Occasionally we could trap more than one soliton and experimentally we have observed situations with $1,2,3$, and 5 permanent solitons on the line. We obtained the most data on the $(2,0)$ step for comparison to the $(1,1)$ step. There was a slightly lower average voltage and a lower resetting threshold for the $(2,0)$ case, consistent with the discussion on the IBM junction (Figs. 5 and 6). However, the overall quantitative differences between the steps were small and we do not consider them further here.

For the steps with more solitons of the same polarity it is very likely that the solitons become bunched-although we cannot tell from the experimental data. This may be illustrated from Fig. 12 which shows a numerical simulation cor-

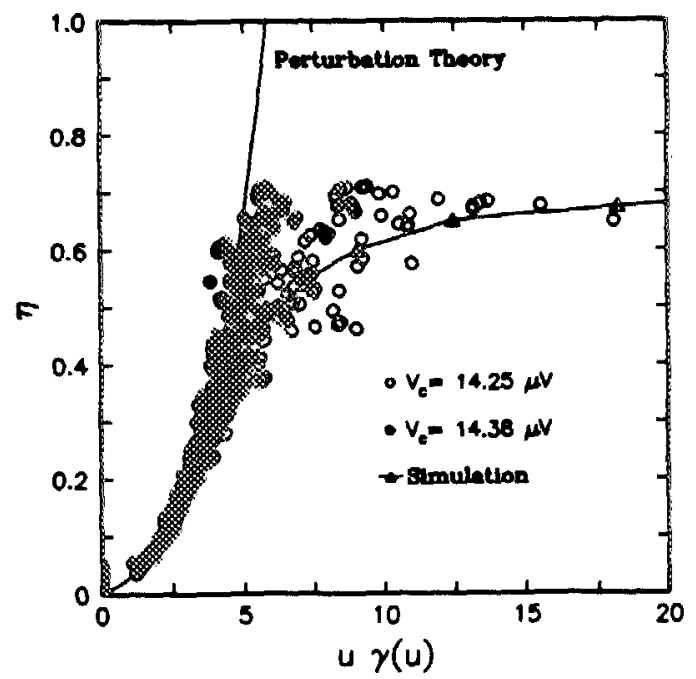

FIG. 10. $\eta$ vs $u \gamma(u)$ curve. The perturbation theory and simulation results used $\alpha=0.02, \beta=0.01$. The closed circles are data fitted to an asymptotic voltage of $14.38 \mu \mathrm{V}$, and the open circles are the same data fitted to 14.25 $\mu \mathrm{V}$.

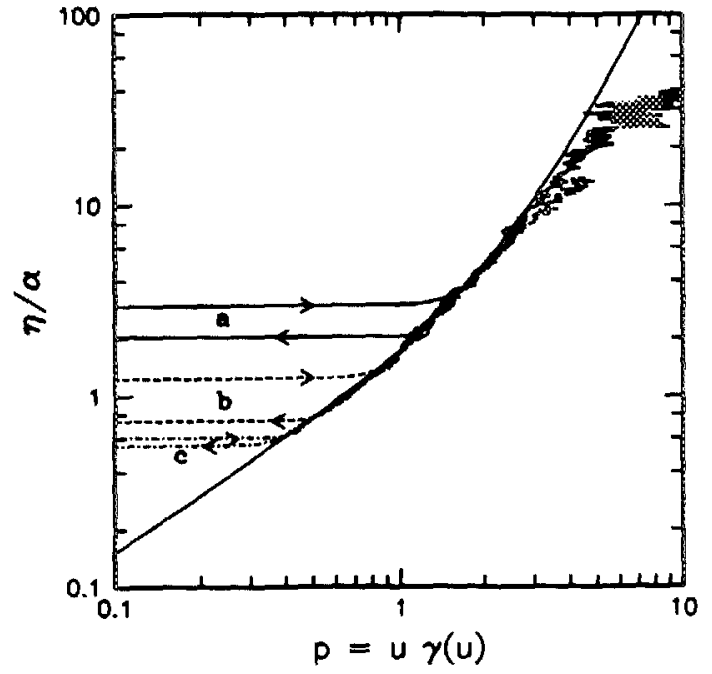

FIG. 11. Smooth line: $\eta / \alpha$ vs $u \gamma(u)$ for $\beta / \alpha=0.556$ [Eq. (5)]. Noisy lines: experimental results for the $(1,0)$ step for three different temperatures between 4.2 and $6.5 \mathrm{~K} . \mathrm{a}, \mathrm{b}$, and $\mathrm{c}$ label the low current behavior of the data in order of descending temperature. At high temperature the hysteresis disappears.

responding approximately to the damping parameters of Fig. 8. Figure 12 shows clearly that bunching takes place, and from the numerical investigation we know that the bunching is an effect of the $\beta$ term. Such bunching has been observed before in numerical simulations ${ }^{13}$ and experiments ${ }^{14}$ though for systems with boundaries.

With trapped solitons on the ring we never observed the ideal situation with zero supercurrent. In the case of one trapped soliton we measured a small ( $\sim 8 \%$ of the soliton free value), double valued supercurrent. The size depended on the history of the junction and was not symmetric with respect to reversed current direction. We explain this by assuming the existence of two pinning sites or potential wells of different depths on the ring. Depending on which well the soliton initially resides in, different forces (bias) are required to initiate the motion and hence different critical currents will be measured. A difference in slopes on the sides of each well could account for the lack of symmetry for re-

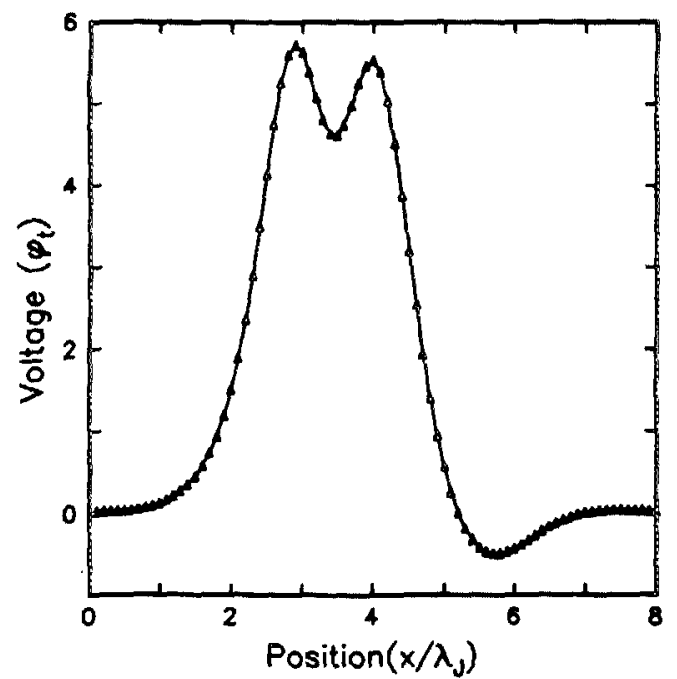

FIG. 12. Numerical simulation of the lineshape for the $(2,0)$ step. $\alpha=0.10$, $\beta=0.05, \eta=0.7$, and $l=8$. Note that bunching has occurred. 
versed current direction. Another indication of pinning has been found for the $(2,0)$ step. In this case the $(1,0)$ step should ideally be absent, but we sometimes observed a small $(1,0)$ step corresponding to one soliton moving and the other at rest in one of the potential wells.

As to the nature of these pinning sites we may consider tunnel barrier imperfections, lithographic imperfections, or an effect intrinsic to the shape of the Lyngby junctions. However, tunnel barrier imperfections would be expected to be small on the scale of the penetration depth in our junctions, and so would lead to a critical current symmetric in polarity. Our observation of asymmetry in the critical current seems to rule out this category. Also, Clem ${ }^{15}$ has estimated the depth of the pinning wells that could be expected just from the symmetry of the junctions: his results are an order of magnitude too small to explain the data. Therefore we judge it most likely that the potential wells are due to unevenness in the lithography: a slow modulation, perhaps, in the width of the junction.

Figure 13(a) displays a direct measurement of $d V / d I$ and its integral for the $(1,0)$ step. The constant of integration was adjusted to fit the directly measured $I-V$ curve. Figure 13(b) plots the same data against $1 / V$. The fine structure, evident in the plots, has also been observed in rectangular junctions, where it is attributed to interactions between soliton motion and plasma oscillations caused by soliton collisions and reflections. ${ }^{10}$ The fact that this fine structure is still present on a junction with neither boundaries nor collisions was not expected, and we must attribute its presence to interactions between the potential wells and the moving solitons. Assuming that the soliton emits plasma waves upon passing through an imperfection, structure in the step is expected whenever the soliton and plasma periods are synchronized. By plotting the values of $1 / V$ (proportional to the soliton period) in Fig. 13(b), taken at the minima, versus an index which is consistent with the estimated plasma frequency, we do indeed find that these fall on a straight line, as shown in Fig. 13(b). Hence, there is strong evidence that the fine structure is caused by interaction with plasma oscillations emitted from the two inhomogeneities on the ring.

We next address the question of soliton stability at high velocities, i.e., the prediction of step heights. Experimentally we have made the observations summarized in Table II. Based on the data sets used in Table I we have computed the critical value of the Lorentz factor $\gamma_{c}$ where switching occurs. By changing the temperature, $\alpha$ and $\beta$ vary over a full decade, but the quantity $D$ of the last column remains constant within $5 \%$. That is,

$$
\alpha \gamma_{c}+(\beta / 3) \gamma_{c}^{3}=D \cong 0.66 \pm 5 \% .
$$

By comparison with Eq. (5) we see that the quantity $D u_{\mathrm{c}}$ is the total damping force when switching occurs. Since $u_{c}$ is always close to one, $D u_{c}$ is also approximately constant (within $15 \%$ ). We propose a qualitative argument to explain why the measured quantity $D$ should be a constant. Since the damping force varies over the width of the soliton, we believe that the soliton is pulled apart by differential drag forces. If we assume that $D$ is proportional to the maximum
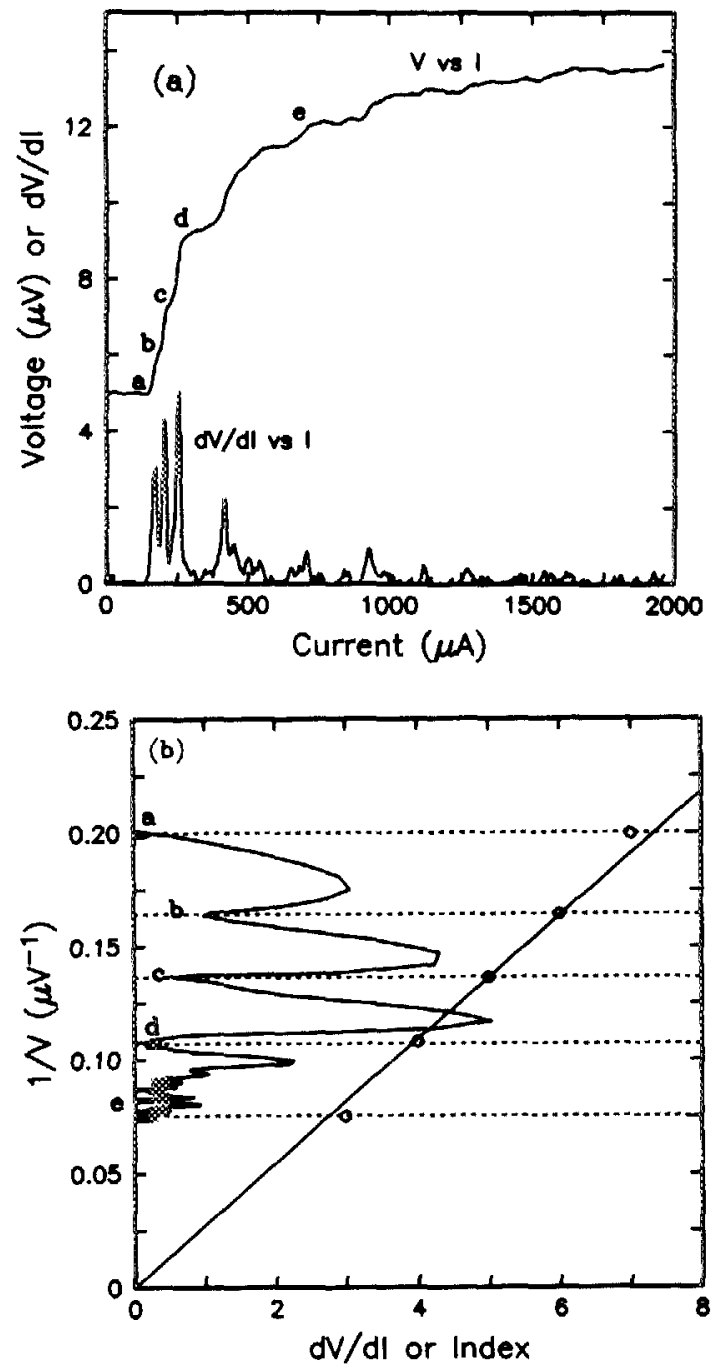

FIG. 13. (a) $d V / d I$ as measured directly, together with the $I-V$ curve obtained by numerical integration of $d V / d I$. The first five minima in the derivative are identified on the integrated curve as a through e. (b ) $d V / d I$ vs $1 /$ $V$ and position of minima vs arbitrary index. (1.0) step, $T=4.2 \mathrm{~K}$. The minima are identified as in (a). The solid line shows the linear behavior of the minima-vs-index plot. The dashed lines show the correspondence between the circles on the index plot and the minima in the derivative.

difference in drag across the width of the soliton, then this would imply that the soliton solution becomes unstable when $D$ exceeds the binding force, $\pi / 4$ in normalized units. We realize that our arguments above are somewhat qualitative and emphasize that the main result is the experimental observation of the constancy of the quantity $D$. We hope this result will provide a clue for further investigations.

TABLE II. Damping parameters showing temperature independence of $D$, the total damping according to perturbation theory. $\gamma_{c}$ refers to the highest value of Lorentz factor, above which the junction switches to the quasiparticle branch of the $I-V$ curve. $\eta_{c}$ is the corresponding maximum normalized bias current.

\begin{tabular}{lllllll}
\hline \hline Data & $\alpha$ & $\beta$ & $\gamma_{c}$ & $\alpha \gamma_{c}$ & $\left\{\beta \gamma_{c}^{3}\right.$ & $\alpha \gamma_{c}+(\beta / 3) \gamma_{c}^{3}$ \\
\hline A352 & 0.018 & 0.01 & 5.59 & 0.1006 & 0.582 & 0.6826 \\
A354 & 0.055 & 0.0306 & 3.64 & 0.2002 & 0.4919 & 0.6921 \\
A355 & 0.008 & 0.0445 & 2.97 & 0.2376 & 0.3886 & 0.6262 \\
A359 & 0.18 & 0.10 & 2.02 & 0.3636 & 0.275 & 0.6386 \\
\hline
\end{tabular}


We want to stress that for high bias (in the vicinity of switching) the mathematics become much more complicated than the simple perturbation theory. We mention a few features that we have found recently by numerical simulation. ${ }^{12}$ (i) In the presence of a $\beta$ term a phase overshoot is created at the trailing edge of the soliton. Eventually as the bias is increased this overshoot creates an extra soliton-antisoliton pair which causes the junction to switch. (ii) In the presence of a $\beta$ term the velocity does not approach 1 asymptotically; rat her the $I-V$ curve is given as a power law for high velocities. The velocity 1 is obtained for the value of bias corresponding to switching.

\section{CONCLUSIONS}

We have experimentally investigated soliton propagation on the annular Josephson junction. We find that our results are well described by perturbation theory if a surface impedance damping term is included. Deviations from perturbation theory are observed for high values of the bias current.

\section{ACKNOWLEDGMENTS}

Stimulating discussions with P. L. Christiansen, $M$. Buettiker, J. Mygind, M. R. Samuelsen, R. D. Parmentier, S. Pagano, and $\mathrm{D}$. Welner are acknowledged. The original sug- gestion for these experiments came from $R$. Landauer and $C$. C. Tsuei.

${ }^{1}$ D. W. McLaughlin and A. C. Scott, Phys. Rev. A 18, 1652 (1978).

${ }^{2} \mathrm{M}$. Buttiker and R. Landauer, Nonlinear Phenomena at Phase Transitions and Instabilities (Plenum, New York, 1982), p. 111.

${ }^{3}$ S. E. Burkov and A. E. Lifsic, Wave Motion 5, 197 (1983).

${ }^{4}$ P. Bodin, N. F. Pedersen, M. R. Samuelsen, and D. Welner, IEEE Trans. Magn. MAG-21, 636 (1985).

${ }^{5}$ N. F. Pedersen, M. R. Samuelsen, and D. Welner, Phys. Rev. B 30, 4057 (1984).

${ }^{6}$ B. Dueholm, A. Davidson, C. C. Tsuei, M. J. Brady, K. H. Brown, A. C. Callegari, M. M. Chen, J. H. Greiner, H. C. Jones, K. K. Kim, A. W. Kleinsasser, H. A. Notarys, G. Proto, R. H. Wang, and T. Yogi, Proceedings of the 17th International Conference on Low Temperature Physics, LT-17, edited by U. Eckern, A. Schmid, W. Weber, and H. Wuhl (Elsevier, New York, 1984).

${ }^{7}$ A. Davidson, B. Dueholm, B. Kryger, and N. F. Pedersen, Phys. Rev. Lett. 55, 2059 (1985).

${ }^{8}$ K. H. Brown, T. Bucelot, A. C. Callegari, M. M. Chen, J. H. Greiner, H. C. Jones, K. K. Kim, A. Kleinsasser, H. A. Notarys, G. Proto, B. J. Van Der Hoeven, R. H. Wang, and T. Yogi (unpublished).

${ }^{9}$ A. Davidson and N. F. Pedersen, Appl. Phys. Lett. 44, 465 (1984).

${ }^{10}$ N. F. Pedersen and D. Welner, Phys. Rev. B 29, 2551 (1984).

${ }^{\prime \prime}$ M. Cirillo, A. M. Cucolo, S. Pace, and B. Savo, J. Low Temp. Phys. 54, 489 (1984).

${ }^{12}$ A. Davidson, N, F. Pedersen, and S. Pagano, Appl. Phys. Lett. 48, 1306 (1986).

${ }^{13}$ P. S. Lomdahl, O. H. Soerensen, and P. L. Christiansen, Phys. Rev. B 25 , 5737 (1982).

${ }^{14}$ B. Dueholm, O. A. Levring, J. Mygind, N. F. Pedersen, O. H. Soerensen, and M. Cirillo, Phys. Rev. Lett. 46, 1299 (1981).

${ }^{15} \mathrm{~J}$. R. Clem (private communication). 